\title{
Neuropsychological Deficits and Sleep in Myotonic Dystrophy
}

\author{
R. Broughton, D. Stuss*, M. Kates, J. Roberts and W. Dunham
}

\begin{abstract}
Eight patients with myotonic dystrophy underwent comprehensive neuropsychological testing and overnight polysomnography to assess whether the waking cognitive impairment might be attributable to sleep structure abnormalities or to sleep-related respiratory problems. Patients showed substantial neuropsychological deficits, fragmented nocturnal sleep and, in half the patients, sleep apnea and/or hypopnea both mainly of central type. There was no statistically significant correlation between the degree of daytime cognitive deficit and the degree of sleep fragmentation or of respiratory problems at night. It was concluded that the neuropsychological deficit in mytonic dystrophy cannot be attributed to a secondary effect of nocturnal sleep apnea or sleep disruption but probably represents a direct effect of CNS lesions.
\end{abstract}

RÉSUMÉ: Déficit neuropsychologique et sommeil dans la dystrophie myotonique Huit malades atteints de dystrophie myotonique ont été étudiés au moyen d'une batterie de tests neuropsychologiques et de la polysomnographie nocturne pour déterminer si le déficit cognitif qui existait pouvait être attribué à une détérioration de la structure du sommeil ou à des difficultés respiratoires au cours du sommeil. On a démontré chez les malades des problèmes neuropsychologiques significatifs de même qu'un sommeil nocturne très fragmenté et, dans la moitié des cas, des apnées et/ou des hypopnées du sommeil, surtout de type central. Il n'existait aucune relation significative entre le degré du déficit cognitif et le degré de la fragmentation du sommeil ou des difficultés respiratoires nocturnes. Nous concluons que le déficit neuropsychologique dans la dystrophie myotonique n'est pas un effet secondaire de la perturbation du sommeil ou de l'apnée nocturne, mais qu'il est probablement une conséquence directe de lésions du système nerveux central.

Can. J. Neurol Sci. 1990; 17: 410-415

Mytonic distrophy is a genetic muscular disorder believed to be inherited as an autosomal dominant trait. As well as the muscular involvement with myotonia (slowness of relaxing contracted skeletal muscle) the condition has been known to be associated with a variety of thalamic and other CNS lesions, neuroendocrine dysfunction, skeletal abnormalities, cardiac problems, testicular atrophy and frontal baldness. In the last few years patients have been found to show varying degrees of neuropsychological deficit. 1,2,3

Diagnostic polysomnograms in myotonic dystrophy have shown various degrees of sleep disturbance and sleep-related respiratory problems which might be the basis of the neuropsychological deficit. To date 35 patients have been reported. Coccagna et al ${ }^{4,5}$ described 6 patients, 2 of whom had significant amounts of central sleep apnea or hypopnea with excessive daytime sleepiness and also exhibited sleep onset REM periods (SOREMPs). Goldenberg and collaborators, ${ }^{6,7}$ on the other hand, recorded 15 patients with 11 having sleep apnea which most frequently (in over $50 \%$ ) was of obstructive type. Of 6 subjects reported by Guilleminault and colleagues, 8.92 had sleep apnea of both obstructive and central types which was associated with daytime sleepiness. Finally, Cirignotta et al ${ }^{10}$ more recently reported 8 further patients, 6 of whom had sleep apnea or hypopnea which in all cases was central in origin.

In none of these studies has cognitive function been assessed. This is surprising in that neuropsychological deficits have been well documented in a number of sleep disorders and especially in apneic patients without myotonic dystrophy. Heavy snoring males, a group known to have a high incidence of obstructive sleep apnea (O.S.A.), have been shown to have neuropsychological deficits compared to controls. 11,12 Patients with O.S.A. whose respiratory indices have been documented during sleep exhibit a global neuropsychological deficit 13,14 which has been correlated with degree of hypoxemia. ${ }^{14}$ Others have found the degree of sleep fragmentation to be a greater predictor of cognitive problems in O.S.A. patients. ${ }^{15}$ O.S.A. patients also have been documented to have both decreased performance on a driving simulator 16 and deteriorated mood, 17 both of which improved on nasal continuous positive airway pressure $(\mathrm{N}$ CPAP). In another study, however, surgical treatment for O.S.A. did not improve mood or cognitive abilities. ${ }^{18}$ The current study was undertaken in order to determine whether the level of daytime neuropsychological function in myotonic dystrophy patients would correlate with the degree of nocturnal sleep disruption

From the Division of Neurology, Ottawa General Hospital and University of Ottawa, Ottawa, Ontario

* Presently at Rotman Research Institute, Baycrest Center, North York, Toronto

Received March I, 1990. Accepted in final form July 31, 1990

Reprint requests to: Dr. R.J. Broughton, Division of Neurology, Ottawa General Hospital, 501 Smyth Road, Ottawa, Canada K1H 8L6 
and/or of sleep-related breathing problems. If so, intervention to improve night sleep quality or sleep-related respiratory difficultics might be expected to improve the cognitive problems from which these patients suffer.

\section{METHODS}

\section{Subjects}

Eight patients ( 5 male, 3 female) aged $15-55$ years (mean 35.6, S.D. 14.4) participated in the study. Their duration of illness ranged from $1-45$ years (mean 17.9, S.D. 14.1). The severity of illness was classified based upon intensity and location of motor weakness following a modified version of the system of Bundy 18 into grade 1 (severe facial and severe limb signs), grade 2 (severe facial and mild limb signs), grade 3 (mild facial and moderate limb signs) and grade 4 (mild facial and mild distal signs). Two subjects (nos. 2,5) were grade 1, 4 subjects (nos. $1,3,4,8$ ) were grade 2,1 subject (no. 3) was grade 3 , and 1 subject (no. 6) was grade 4 . None of the patients was receiving active CNS medication at the time of the study. All underwent overnight polysomnography and a comprehensive neuropsychological examination.

\section{Polysomnography}

Overnight polysomnography included EEG (Fp2-A1, C4-A1, O2-A1, Fp1-A2, C3-A2, O1-A2), right and left electro-oculogram (outer canthus to contralateral earlobe), submental EMG, upper airway air exchange (by thermocouple) from the right and left nostrils and mouth, chest and abdominal respiration (by Respitrace), right and left anterior tibialis EMGs and, in 4 patients, continuous transcutaneous $\mathrm{pO}_{2}$ monitoring (Novametrix model 818 apparatus) the accuracy of which was controlled by blood gas determinations. Sleep stages were visually scored by Rechtschaffen-Kales criteria. ${ }^{19}$

The sleep structure variables $(\mathrm{N}=23)$ were: total sleep period (TSP) from sleep onset to morning awakening (min.); total sleep time (TST) with and without stage I ( $\mathrm{min}$.); sleep period efficiency with and without stage $1(\%)$; amount (min.) and percent of TSP in wakefulness after sleep onset (WASO), stages 1 , 2, 3, 4 and REM sleep; numbers of REM periods, awakenings lasting over 1 minute and arousals (with alpha or EEG desynchronization) lasting 10 seconds or more; and latencies (min.) to sleep onset, SWS (initial stage 3 ) and REM sleep.

The numbers of central, mixed or obstructive apneas as well as of hypopneas $>10 \mathrm{sec}$ in duration were scored. During obstructive apneas total upper airway flow (thermocouple) ceased with continuation of respiratory effort and frequent paradoxical breathing between chest and abdomen (Respitrace). In central apneas both upper airway airflow and respiratory effort ceased. Mixed apneas were defined as a succession of central and obstructive patterns within the same apneic event. In hypopneas upper airway flow was transitorily reduced by more than $50 \%$ from immediately preceding baseline levels. In obstructive hypopnea respiratory effort continued, whereas in central hypopneas it was similarly reduced by more than $50 \%$. The criteria are those in Guilleminault. ${ }^{20}$ The respiratory variables $(\mathrm{N}=8)$ included: number $>10 \mathrm{sec}$ in duration of central, mixed and obstructive apneas, number of hypopneas, apnea index (no. apneas/hr. of sleep), apnea + hypopnea index (no. apneas + hypopneas/hr. of sleep), total apnea + hypopnea time (min.), and duration (min.) of hypoxemia with $\mathrm{pO}_{2}<60 \mathrm{~mm} \mathrm{Hg}$.

\section{Neuropsychological Testing}

An extended neuropsychological test battery was administered in the original study reported elsewhere. ${ }^{3}$ The tests in the present project were selected to provide a sampling of pertinent functions such as overall intelligence, memory, different aspects of attention, speed of information processing and verbal flucncy. Neuropsychological tests were administered by trained neuropsychologists (DS, MK) and included the Wechsler Adult Intelligence Scale - Revised (WAIS-R);21 the Trail Making Test; 22 the Stroop test; ${ }^{23}$ the F-A-S test of word list generation 24 and the Wechsler Memory Scale. ${ }^{25}$ To minimize fatigue in these handicapped patients, testing was normally completed in two sessions with 2-3 days between sessions. Each session lasted 2-3 hours and had adequate rest pauses.

The WAIS-R is a general test battery with a number of subtests sampling multiple psychological functions. A prorated full scale IQ excluding the subtests of Comprehension, Picture Completion, Picture Arrangement and Digit Symbol was the dependent measure used.

The Digit Span is a subtest of the WAIS-R which comprises two subtests, Digit Forwards and Digit Backwards. Dependent measures were number of digits correctly answered forwards and backwards, and total number.

The Trail Making Test (TMT) is a visuo-motor tracking lask requiring the subject to join as rapidly as possible by pen randomly located numbers in numerical sequence (part A) or in alternate numerical-alphabetical sequence (part B). The time in seconds required to complete each section $A$ and $B$ and total time were the dependent measures.

The Stroop test is a test of attention, concentration, and reading fluency. Subjects are presented with a card containing rows of three colour words (e.g., red, green, blue) printed in black and white, a card with colour patches (no words) arranged similarly to the "word" card, and then a combined word-colour interference card. With the first card the subject is requested to read the words row by row as rapidly as possible. With the second card the subject is asked to name the colours as quickly as possible. With the third (interference) card the subject is told that the three colour words will be presented in a different colour than the named colour and is requested to give the actual colour rather than the named colour as rapidly as possible. Dependent measures were the time in seconds to complete each of the three cards.

The $\boldsymbol{F}$-A $-\mathrm{S}$ test is one of language ability and speech production. The subject is asked to produce words beginning with the letter " $F$ ", excluding names of people or places, for a duration of 60 seconds. This is repeated for the letters " $A$ " and " $S$ ". The dependent measure is the total production of words across the three letters.

The Wechsler Memory Scale is a comprehensive memory test consisting of 7 subtests involving orientation, mental control, memory of stories, associate learning and other functions. The dependent measures analyzed included a mental control score and the derived Memory Quotient.

\section{Statistics}

In order to determine whether the neuropsychological capabilities could be secondary to sleep structure abnormalitics or to 
respiratory problems in sleep, each performance variable was correlated with each sleep variable by bivariate Pearson produce moment correlations. ${ }^{28}$ A total of 434 correlations were performed.

\section{RESUlTS}

Sleep

Nocturnal sleep structure results are summarized in Tables 1 and 2 . It can be seen that myotonic dystrophy patients are very variable in their sleep architecture. As a group they showed overall long sleep latencies, high amounts of wakefulness after sleep onset, low sleep efficiencies, low amounts of stage 2 and REM sleep, reduced numbers of REM periods, and relatively normal amounts of SWS.

Their respiratory measures (Table 3 ) were also highly variable. Three patients had an apnea index over 10; and 4 had an apnea + hypopnea index over 20 . Essentially all of the sleeprelated respiratory events were of central type, only patient 3

\begin{tabular}{|c|c|c|c|c|c|c|c|c|c|c|c|c|c|c|}
\hline Patient & Sex & Age & Dur'n. & $\begin{array}{c}\text { TSP } \\
\text { (min.) }\end{array}$ & $\begin{array}{c}\text { TST } \\
+ \text { St } 1 \\
\text { (min.) } \\
\end{array}$ & $\begin{array}{c}\text { TST } \\
- \text { St } 1 \\
\text { (min.) }\end{array}$ & $\begin{array}{l}\text { Eff'y. } \\
+ \text { St } 1 \\
(\%)\end{array}$ & $\begin{array}{c}\text { Eff'y. } \\
-\mathrm{St} 1 \\
(\%)\end{array}$ & $\begin{array}{c}\text { Sleep } \\
\text { lat. } \\
\text { (min.) }\end{array}$ & $\begin{array}{c}\text { SwS } \\
\text { lat. } \\
\text { (min.) }\end{array}$ & $\begin{array}{c}\text { REM } \\
\text { lat. } \\
\text { (min.) }\end{array}$ & $\begin{array}{c}\text { REMPs } \\
\text { (no.) }\end{array}$ & $\begin{array}{c}\text { Wakes } \\
1>\text { min. } \\
\text { (no.) }\end{array}$ & $\begin{array}{c}\text { Arousals } \\
\text { (no.) }\end{array}$ \\
\hline 1 & $\mathbf{M}$ & 15 & 1 & 582.3 & 530.3 & 475.7 & 91.1 & 81.7 & 15.7 & 5.3 & 202.3 & 3 & 3 & 15 \\
\hline 2 & M & 26 & 11 & 448.0 & 174.0 & 163.5 & 38.8 & 36.5 & 72.0 & 5.5 & 236.0 & 2 & 4 & 24 \\
\hline 3 & $M$ & 30 & 15 & 435.2 & 345.2 & 249.3 & 86.6 & 62.6 & 36.5 & 14.5 & 187.2 & 2 & 5 & 164 \\
\hline 4 & M & 40 & 31 & 446.3 & 351.7 & 236.0 & 78.8 & 52.9 & 36.3 & 9.7 & 140.7 & 4 & 4 & 9 \\
\hline 5 & $M$ & 55 & 45 & 439.3 & 313.7 & 240.0 & 71.4 & 54.6 & 58.0 & 18.3 & 113.7 & 2 & 5 & 0 \\
\hline 6 & $\mathrm{~F}$ & 23 & 8 & 478.5 & 369.0 & 328.0 & 77.0 & 68.4 & 16.0 & 11.0 & 97.0 & 2 & 3 & 2 \\
\hline 7 & $\mathrm{~F}$ & 44 & 20 & 358.0 & 260.0 & 226.7 & 72.6 & 63.3 & 26.7 & 3.3 & 246.3 & 1 & 4 & 0 \\
\hline 8 & $\mathrm{~F}$ & 52 & 12 & 355.0 & 135.5 & 80.0 & 38.2 & 22.5 & 68.0 & 6.5 & 0 & 0 & 2 & 145 \\
\hline $\bar{x}$ & & 35.6 & 17.9 & 443.0 & 309.9 & 250.0 & 68.4 & 59.7 & 41.4 & 9.4 & 152.9 & 2.0 & 4.0 & 45 \\
\hline SD & & 14.4 & 14.1 & 71.5 & 123.3 & 116.0 & 19.4 & 17.2 & 22.3 & 5.4 & 82.3 & 1.2 & 1.0 & 68 \\
\hline
\end{tabular}

TSP = total sleep period; TST + St I = total sleep time including stage I (drowsiness); TST - St I = total sleep time without stage I; Eff ' $y .=$ sleep efficiency; lat. = latency: REMPs = REM sleep periods; wakes = awakenings after sleep onset; arousals = EEG signs of arousal.

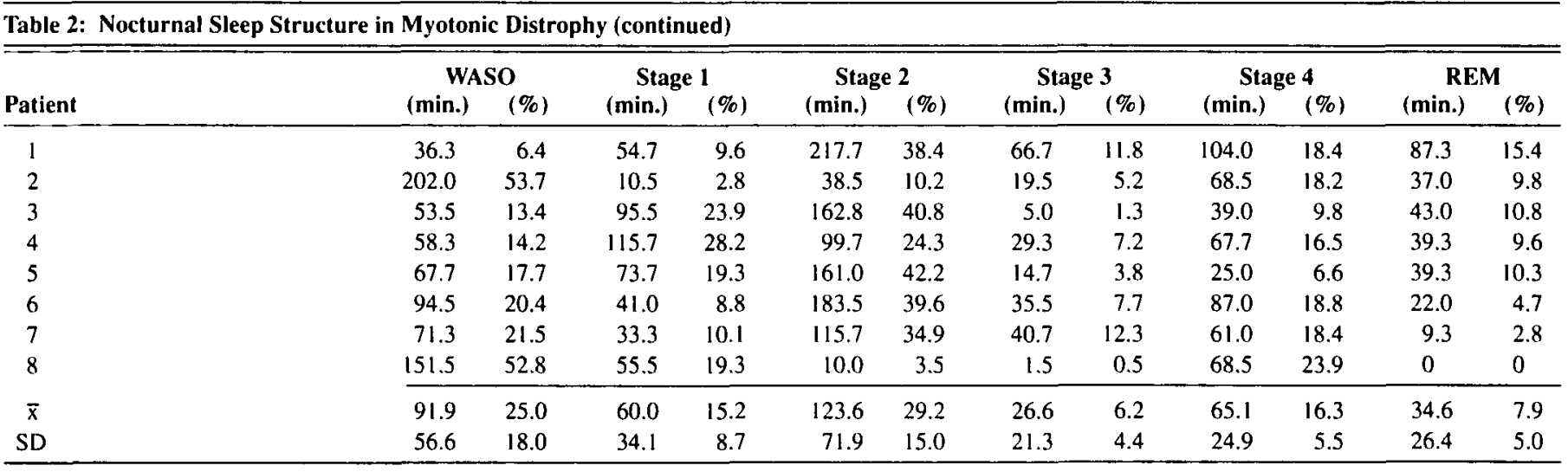

WASO $=$ wakefulness after sleep onset

\begin{tabular}{|c|c|c|c|c|c|c|c|c|}
\hline \multirow[b]{2}{*}{ Patient } & \multicolumn{3}{|c|}{ Apneas } & \multirow[b]{2}{*}{$\begin{array}{c}\text { Hypop. } \\
\text { (no.) }\end{array}$} & \multirow{2}{*}{$\begin{array}{c}\text { Apnea } \\
\text { Index } \\
(/ \mathrm{hr} .)\end{array}$} & \multirow{2}{*}{$\begin{array}{c}\text { Ap. + Hyp. } \\
\text { Index } \\
(/ \mathrm{hr} .)\end{array}$} & \multirow{2}{*}{$\begin{array}{l}\text { Ap. + Hyp. } \\
\text { Dur'n. } \\
\text { (min.) }\end{array}$} & \multirow{2}{*}{$\begin{array}{c}\text { Hypoxem. } \\
\text { pO2 } 60 \\
\text { (min.) }\end{array}$} \\
\hline & $\begin{array}{l}\text { Cent. } \\
\text { (no.) }\end{array}$ & $\begin{array}{c}\text { Mixed } \\
\text { (no.) }\end{array}$ & $\begin{array}{l}\text { Obs. } \\
\text { (no.) }\end{array}$ & & & & & \\
\hline 1 & 34 & 0 & 0 & 164 & 9.5 & 28.1 & 60.0 & 9.0 \\
\hline 2 & 21 & 0 & 0 & 72 & 7.2 & 32.1 & 25.0 & NR \\
\hline 3 & 250 & 7 & 10 & 153 & 46.4 & 73.0 & 131.5 & 361.0 \\
\hline 4 & 16 & 0 & 0 & 16 & 2.7 & 5.5 & 7.8 & $N R$ \\
\hline 7 & 0 & 0 & 0 & 0 & 0 & 0 & 0 & $\mathrm{NR}$ \\
\hline 8 & 199 & 0 & 0 & 115 & 88.1 & 139.0 & 81.9 & NR \\
\hline $\bar{x}$ & 73 & 1.0 & 1.0 & 68.0 & 19.6 & 35.6 & 39.5 & 114.0 \\
\hline SD & 98 & 2.5 & 3.5 & 67.7 & 31.6 & 48.4 & 47.6 & 168.3 \\
\hline
\end{tabular}

Cent. = central apnea, obs. = obstructive apnea, Hypop. = hypopneas, Ap. + Hyp. = apnea plus hypopnea, NR = not recorded. All patients had exclusively central hypopneas except patient 3 who had 141 central and 12 obstructive hypopneas. 
showing a small proportion of obstructive events. More than 30 minutes of significant hypoxemia $\left(\mathrm{pO}_{2}<60 \mathrm{~mm} \mathrm{Hg}\right.$ ) was present in 2 of the 4 patients monitored by transcutaneous oximetry. In all 4 blood gas levels confirmed accuracy of the transcutaneous monitor.

Sleep histogram examples are shown of a patient (no. 3) with marked apnea and hypopnea mainly of central type (Figure 1) and of a patient (no. 5) with substantial sleep fragmentation but little respiratory difficulty in sleep (Figure 2).

\section{Neuropsychological Tests}

The results of the neuropsychological testing for the 14 variables derived from the tests are summarized in Table 4. Again, considerable intersubject variability is evident. An IQ score on

Table 4: Neuropsychological Test Results in Patients with Myotonic Distrophy

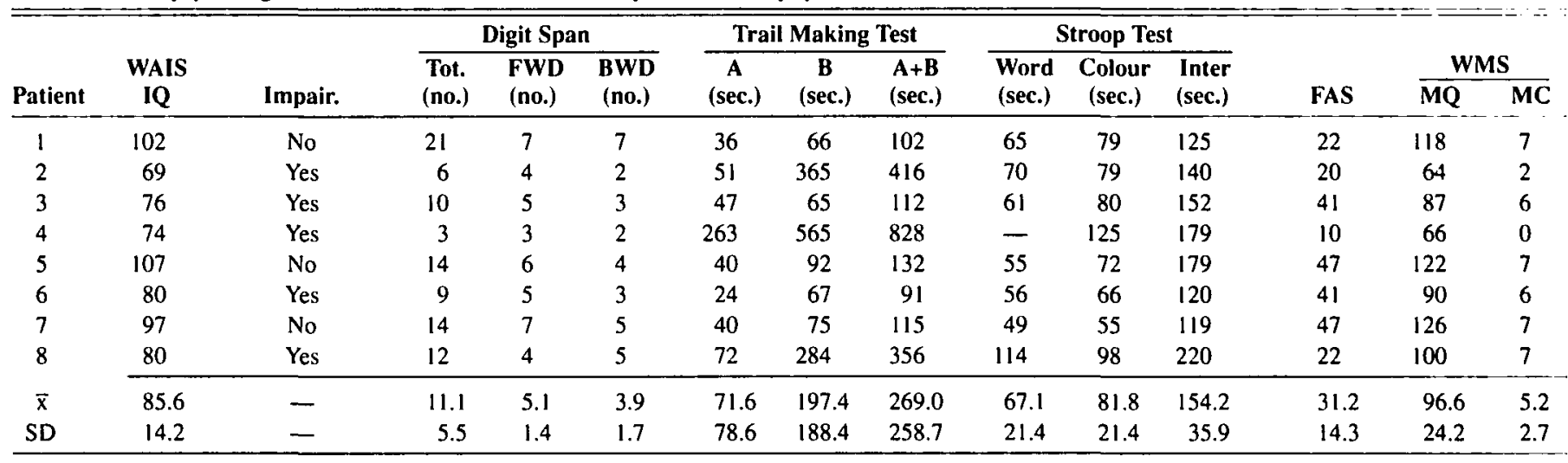

$I Q=$ Intelligence Quotient on the WAIS-R; Tot. = total raw score; FWD and BWD = longest span recalled correctly in forwards and backwards order on the Digit Span; A, B and A + B are the test scores on the Trail Making Test; Inter. = interference; FAS = F-A-S test; WMS = Wechsler Memory Scale; MQ = Memory Quotient; $M C=$ mental control measure.

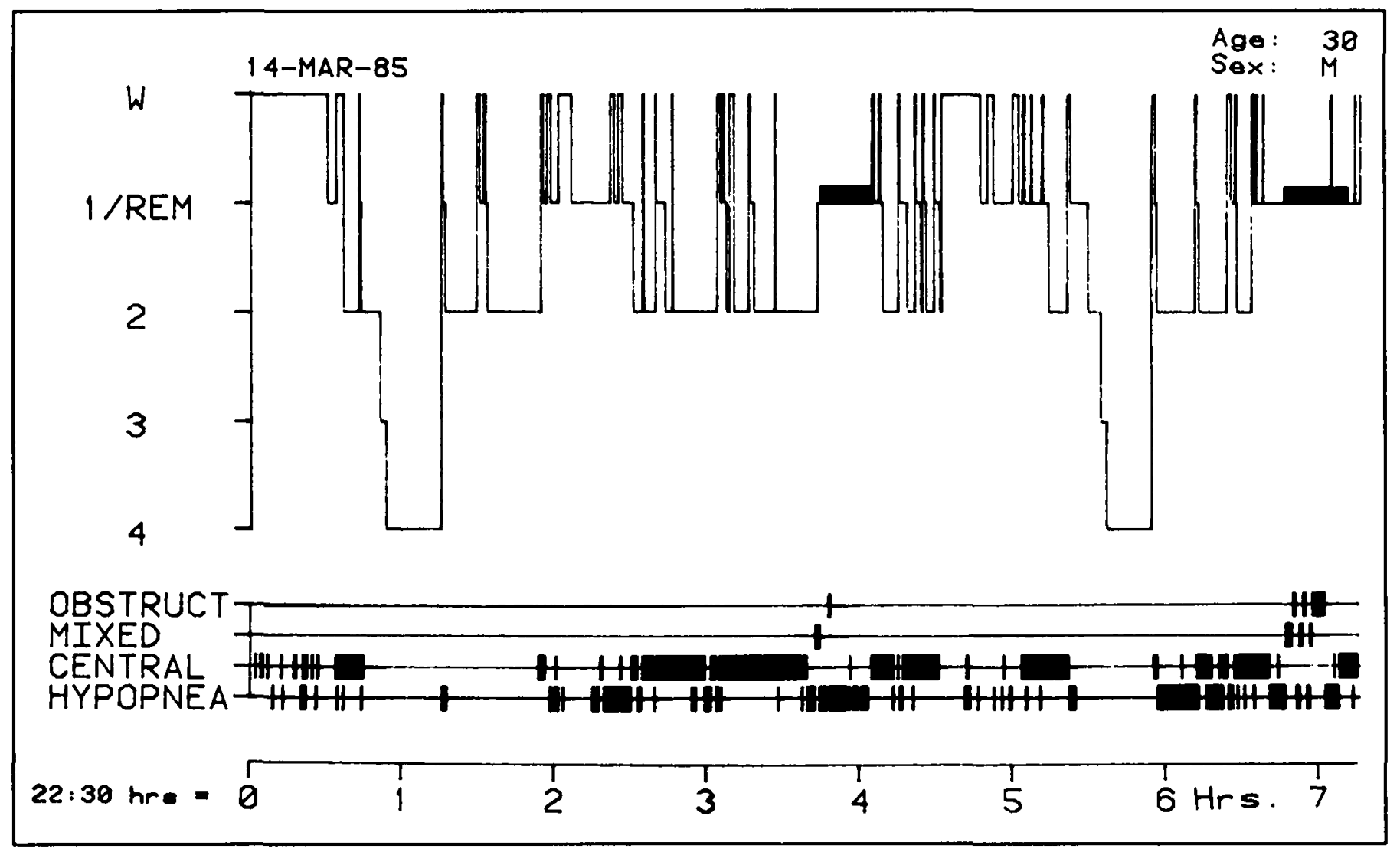

Figure I - Sleep histogram of a 30-year-old male myotonic dystrophy patient with fragmented sleep and marked sleep apnea and hypopnea, mainly central in type. (Each vertical line in the respiratory measures indicates presence of I or more apneas of hypopneas during that I minute cpoque.) 


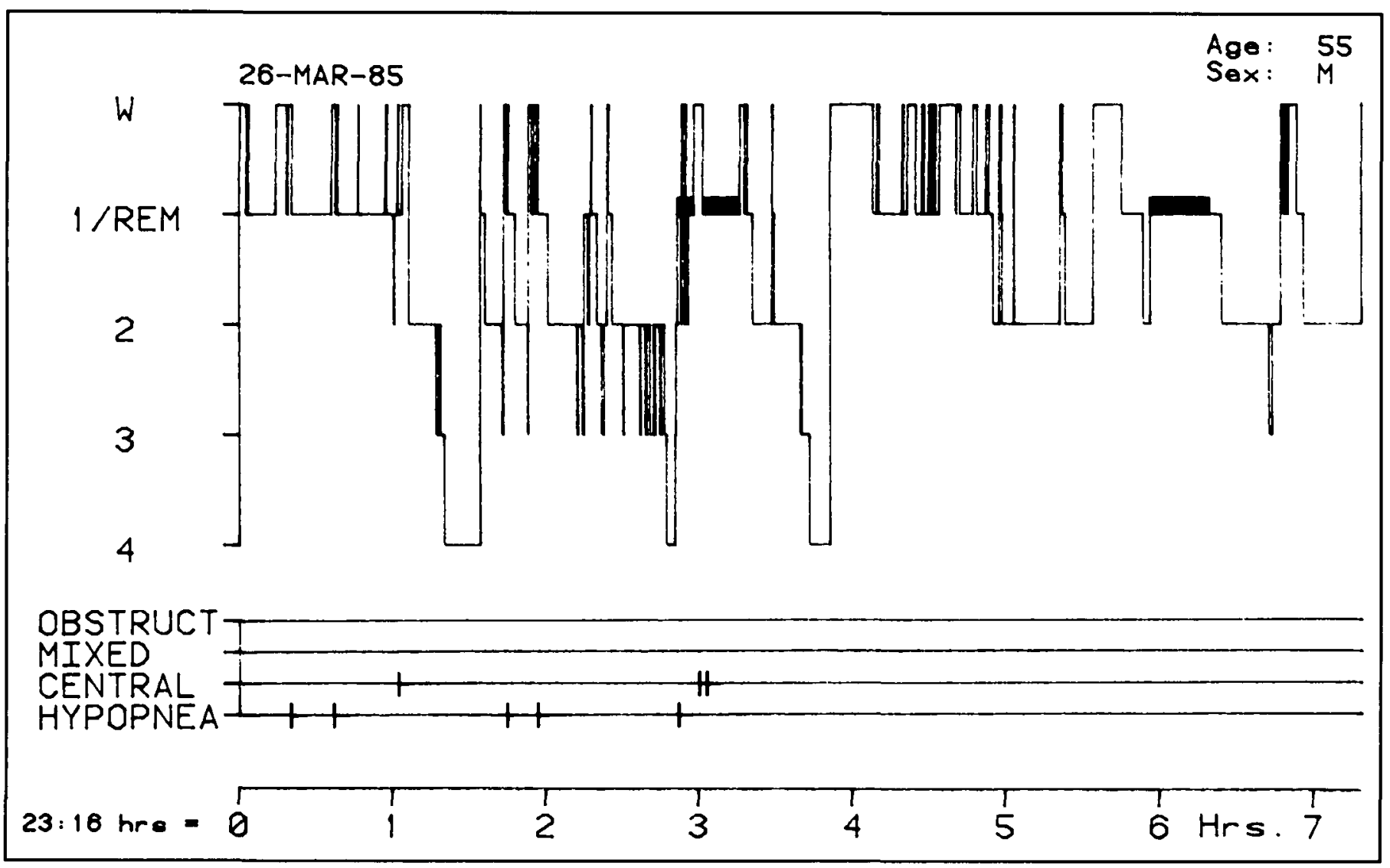

Figure 2 - Sleep histogram of a 55-year-old male myotonic dystrophy patient showing substantial sleep fragmentation unassociated with significant amounts of sleep-related respiratory problems.

the WAIS-R of less than 80 was chosen as the criterion for significant cognitive impairment. This indicated that 5 subjects were impaired and 3 were not.

\section{Correlations}

Of the 434 Pearson product moment correlations tested only 2 were significant at $p<.01$. The number of words on the Stroop test correlated positively with the apnea index $(r=.87$, $\mathrm{p}=<.005)$ and apnea + hypopnea index $(\mathrm{r}=.91, \mathrm{p}=<.002)$. These correlations would suggest higher performance on this single measure with greater sleep-related respiratory difficulties. However, at a $1 \%$ confidence level at least 4 of the 434 correlations would be expected to be significant by chance. It is therefore apparent no significance can be attributed to the two correlations found. Because of sample size, no attempt was made to control for possible effects of age and eduction.

\section{Discussion}

Sleep structure in myotonic dystrophy patients was found to be disrupted and abnormal. It was characterized by sleep fragmentation, short REM latencies and reduced REM amounts. These results largely confirmed those of others who have reported mostly low sleep efficiencies, high amounts of wakefulness after sleep onset, normal amounts of SWS and occasional sleep onset REM periods. 4.5 .6 .8

Respiratory problems in sleep were mainly central in type. These findings support those of the Bologna group.4.5.10 Others have reported a predominance of obstructive over central sleeprelated respiratory events. $6.7,8.9 \mathrm{lt}$ is therefore evident that both main types of respiratory problems can occur in myotonic dystrophy. The obstructive events may in fact also be neural in origin, due to inadequate CNS control of upper airway muscle tone. ${ }^{8}$ Unlike for obstructive sleep apneic patients without myotonic dystrophy, there was no strong correlation between degree of sleep disruption and the magnitude of respiratory problems in sleep. It is possible that CNS lesions, as well as muscular or respiratory causes, might be involved in the creation of their sleep fragmentation. Thalamic lesions have been shown to cause organic insomnia;27 and cytoplasmic eosinophilic infiltrations have been documented in this subcortical area in myotonic dystrophy. ${ }^{28}$ Two of our patients had severe nocturnal hypoxemia, which has also been described for myotonic dystrophy patients by Coccagna et al ${ }^{4}$ and Guilleminault et al. ${ }^{8}$

The neuropsychological deficits found in this study support the cognitive difficulties described by others using similar tests. 1.2.3 Myotonic dystrophy patients as a group certainly perform normal-average. Yet, we found a wide range of cognitive abilities similar to the high interindividual variability in sleep and nocturnal respiratory measures.

This is the first study to compare neuropsychological status with sleep variables in patients with myotonic dystrophy. Our major hypothesis that daytime cognitive function would reflect the degree of night sleep disturbance and/or of nocturnal respiratory problems was disconfirmed by the results. The 5 patients 
with significant cognitive deficit by WAIS-R were not necessarily those with greatest sleep disturbance or breathing problems in sleep. This result indicates that the sleep and cognitive difficulties in myotonic dystrophy are probably independent features of the disease. Perhaps each is related to lesions and/or dysfunction in different brain areas which vary from patient to patient. Because of this independence, treatment of sleep quality or of sleep-related respiratory problems would not be anticipated to lead to significant improvement in neuropsychological function in this patient group, although this remains to be directly tested.

\section{ACKNOWLEDGEMENTS}

This project was supported by a grant from the Medical Research Council of Canada, of which one author (R.B.) is a Career Investigator Awardee and by a research grant of the Ontario Mental Health Foundation to another (D.S.). Barbara Reynolds kindly provided secrelarial assistance.

\section{REFERENCES}

1. Woodward JB, Heaton RK, Simon DB, et al. Neuropsychological findings in myotonic dystrophy. J Clin Neuropsycho $1982 ; 4: 335-342$

2. Bird TD, Follett C. Griep E. Cognitive and personality function in myotonic dystrophy. J Neurol Neurosurg Psychiatry 1983; 46: 971-980.

3. Stuss D, Kates MH, Poirier CA, et al. Evaluation of information processing speed and neuropsychological functioning in patients with myotonic dystrophy. J Clin Exp Neuropsychol 1987; 9 : 131-146.

4. Coccagna G, Marinelli P, Lugaresi E. Sleep and alveolar hypoventilation in myotonic dystrophy. Acta Neurol Belg 1982; 82: 185-194.

5. Coccagna G, Mantovani M, Parchi C, et al. Alveolar hypoventilation and hypersomnia in myotonic dystrophy. J Neurol Neurosurg Psychiatry 1975; 38: 977-984.

6. Goldenberg F, Perrier M, Harf A, et al. Sleep and respiratory patterns in myotonic dystrophy. In: Koella WP, Levin P, eds. Sleep 1976. Basel: Karger 1977; 439-442.

7. Leygonie-Goldenberg F, Perrier M, Duizabo PL, et al. Troubles de la vigilance, du sommeil et de la fonction respiratoire dans la maladie de Steinert. Rev Neurol (Paris) 1977; 133: 255-270.

8. Guilleminault C. Cummiskey J, Motta J, et al. Respiratory and hemodynamic study during wakefulness and sleep in myotonic dystrophy. Sleep 1978; 1: 19-31.

9. Cummiskey J, Lynne-Davies P, Guilleminault C. Sleep study and respiratory function in myotonic dystrophy. $\ln$ : Guilleminault $\mathrm{C}$, Dement W, eds. Sleep Apnea Syndromes. New York: Liss 1978; 295-308.

10. Cirignotta F, Mondini S, Zuccini M, et al. Sleep-related breathing impairment in myotonic dystrophy. J Neurol 1987; 235: 80-85.
11. Berry DT, Webb WB, Block AJ, et al. Nocturnal hypoxial and neuropsychological variables. J Clin Exp Neuropsychol 1986; 8: 229-238.

12. Telakivi T, Kajaste $S$, Partinen $M$, et al. Cognitive function in middle-aged snorers and controls: roles of excessive daytime somnolence and sleep-related hypoxic events. Sleep 1988; 11: 454-462.

13. Kales A, Caldwell AB, Cadieux RJ, et al. Severe obstructive sleep apnea - II: associated psychopathology and psychosocial consequences. J Chron Dis $1985 ; 38$ : 427-434.

14. Greenberg GD, Watson RK, Deptula D. Neuropsychological dysfunction in sleep apnea. Sleep 1987; 10: 254-262.

15. Besset $A$. Tafti M, Billiard $M$. Is cognitive impairment in OSAS related to cerebral hypoxia or to sleep fragmentation? Presented at the 10th Congress of the European Sleep Research Socicty. Strasbourg, France, May 1990.

16. Findlay LJ, Fabrizio MJ, Knight H, et al. Driving simulator performance in patients with sleep apnea. Am Rev Respir Dis 1989; 140: $529-530$.

17. Derderian SS, Bridenbaugh RJ, Rajogopal KR. Neuropsychological symptoms in obstructive sleep apnea improve after treatment with nasal continuous positive airway pressure. Chest 1988; 94: 1023-1027.

18. Bundy S. Clinical evidence for heterogenecity in myotonic dystrophy. J Med Genet 1982; 19: 341-348.

19. Rechischaffen A, Kales A, eds. A Manual of Standardized Terminology, Techniques and Scoring Systems for Sleep Stages of Human Subjects. Washington, D.C.: National Institutes of Health Publication 1968; no. 204.

20. Guilleminault $C$, ed. Sleeping and Waking Disorders: Indications and Techniques. Menlo Park. CA: Addison-Wesley 1982.

21. Wechsler D. WAIS-R Manual. Wechsler Adult Intelligence Scale Revised. New York: Harcourt Brace Jovanovich 1981.

22. Army Individual Test Battery. Manual of directions and scoring. Washington, D.C.: U.S. Army War Department, Adjutant General's Office.

23. Stroop JR. Studies of interference in serial verbal reactions. J Exp Psychol 1935; 18: 643-662.

24. Spreen O, Benton AL. Neurosensory Center comprehensive examination for aphasia. Victoria, B.C.: Neuropsychology Laboratory, Depanment of Psychology, University of Victoria.

25. Wechsler, D. A standardized memory scale for clinical use. J Psychol 1945; 19:87-95.

26. Glass GV, Stanley JC. Statistical methods in education and psychology. Englewood Cliff, N.J.: Prentice-Hall 1970.

27. Lugaresi E, Medori $R$, Montagna $P$, et al. Fatal insomnia and dysautonomia with selective degeneration of thalamic nuclei. $N$ Engl J Med 1986; 315: 997-1003.

28. Culebras A, Feldman RG, Merk FB. Cytoplasmic inclusion bodies within neurones of the thalamus in myotonic dystrophy: a light and electron microscope study. J Neurol Sci 1973; 19: 319-329. 